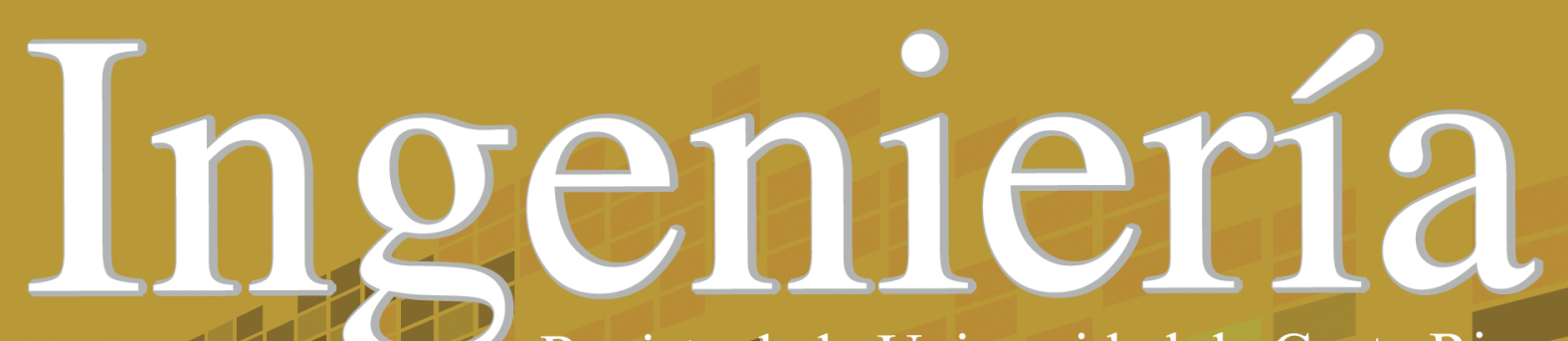

Revista de la Universidad de Costa Riea ENERO/JULIO 2013 - VOLUMEN 23 Número (1)

H

H

H71

Tr.

$x^{2}$

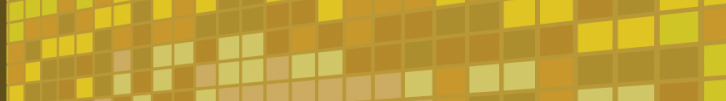

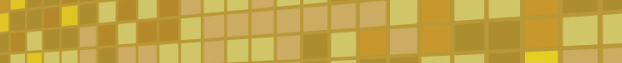

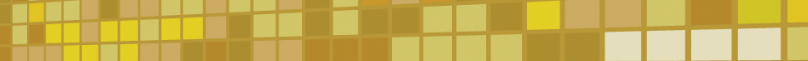

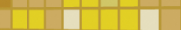

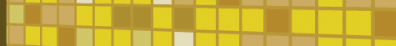

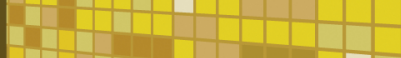

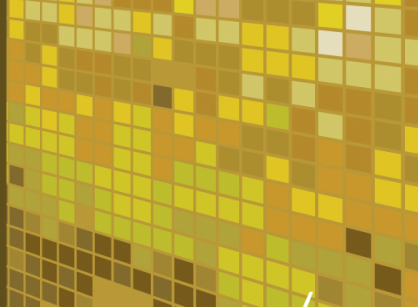

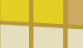



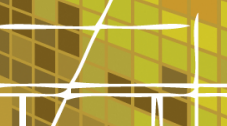

$+\infty \square$ 


\title{
COMPORTAMIENTO EN CORTANTE DE MUROS DE MAMPOSTERÍA CON EL REFUERZO EMBEBIDO EN CONCRETO COLOCADO EN LAS SISAS DE LOS BLOQUES
}

\begin{abstract}
Resumen
Se presenta un estudio sobre el comportamiento de muros de mampostería integral sometidos a cargas paralelas a su plano, utilizando dos tipos de configuraciones de refuerzo a cortante, a) acero horizontal colocado directamente sobre las sisas de los bloques y b) totalmente embebido en concreto de relleno. Se compara el comportamiento de ambos tipos de reforzamiento y se analizan las diferencias entre los valores de resistencia teórica en cortante calculados según los códigos sísmicos de Costa Rica (CSCR-02 y CSCR-10).

Los ensayos consistieron en la aplicación de cargas paralelas al plano para ambos tipos de muros. Se utilizaron dos especímenes de cada configuración a escala natural equivalentes en cuanto a cantidad de refuerzo, tipo de bloques, altura y longitud.

$\mathrm{Al}$ analizarse los resultados obtenidos se encontró que los muros con el refuerzo en las sisas presentaron menores valores de rigidez, y su agrietamiento comenzó a cargas menores que en los muros con el acero embebido. En el rango inelástico ambos tipos de muros presentaron valores similares de disipación de energía.

Se pudo determinar que no existe diferencia significativa en la resistencia a cortante entre los muros al utilizar el acero horizontal entre las sisas o embebido en concreto, y que los cálculos teóricos tienden a ser muy conservadores. Sin embargo, hay que hacer notar que en este estudio no se evaluaron los empalmes en varillas horizontales.
\end{abstract}

Palabras clave: Muros de mampostería; escala natural; resistencia a cortante.

\section{Summary}

The shear behavior of masonry walls tested for in plane loads using two horizontal reinforcement configurations, a- with the reinforcement settled in the mortar beds and b-fully embedded in grout, is presented. The behavior of both types of reinforcement were evaluated, and the differences between the strength values given by the Costa Rican seismic codes in its latest versions 2002 and 2010 (CSCR-02 and CSCR-10) were calculated and compared with the experimental values.

Four experimental walls were tested with in plane lateral loads. Two full scale specimens for each reinforcement configuration were used. Each configuration was equivalent in amount of reinforcement, block type, height and length.

After calculating the theoretical strengths, it was observed that the walls with the reinforcement laid in the mortar beds presented lower stiffness values, and cracking began at smaller loads than in the walls with the reinforcement fully embedded. In the inelastic range, both types of walls showed similar values of energy dissipation.

The strengths calculated with CSCR-10 for both types of walls, were lower than the ones calculated with CSCR02. On the other hand, the results from the tests showed no significant differences between the two types of walls, and these results were significantly higher than the theoretical ones. However, it is important to point out, that horizontal splices were not evaluated in this study.

Key words: Masonry walls; full size specimens; shear strength. 


\section{INTRODUCCIÓN}

Actualmente en Costa Rica, el método de construcción más utilizado para viviendas y edificaciones de baja y mediana altura es la mampostería. Este hecho se debe principalmente a que es un sistema fácil de construir y económico.

A pesar de la popularidad de la mampostería en el campo de la construcción, sus propiedades mecánicas según nuestras prácticas constructivas aún no han sido totalmente establecidas. Esto debido a la variedad de materiales que conforman estos sistemas estructurales y a la variabilidad de sus propiedades debido al proceso constructivo.

A nivel internacional a partir de diversas investigaciones, el comportamiento de la mampostería ante cargas sísmicas ha sido estudiado de distintas formas para facilitar su comprensión. Sin embargo, en todos estos estudios, se evalúan las prácticas constructivas con metodologías diferentes a las nuestras, por lo que se debe generar investigación propia que tome en cuenta estas diferencias.

Por esta razón el tema de la adherencia del acero horizontal es de suma importancia, para garantizar la resistencia requerida en cortante. Debido a esto, en el CSCR-10 se implementó un cambio en el cálculo de la capacidad en cortante de los muros, donde se considera si el acero se coloca embebido en concreto de relleno, o en las sisas de los bloques.
En el Cuadro 1 se observan las diferencias en el cálculo de la resistencia en cortante de muros de mampostería de acuerdo a la colocación del acero horizontal. Se puede apreciar una disminución significativa en la contribución del acero horizontal entre ambos códigos, dependiendo de si el acero está embebido en concreto de relleno o colocado sobre las sisas.

A nivel nacional, el tema de la mampostería ha sido estudiado en diferentes investigaciones, las más relevantes respecto a este estudio son: Gamboa (1997), Venegas (1997), Sandi (1998), Hidalgo (2005).

A pesar de que se han encontrado datos muy relevantes tales como la importancia del refuerzo horizontal en los muros y la importancia de la adherencia de este acero, no se tienen resultados concluyentes a partir de ensayos a escala natural, sobre la colocación del mismo y su influencia en la resistencia a cortante.

A partir de lo anterior, con base en los cambios dados en el CSCR-10 y en los datos que se tienen de investigaciones, y siendo la mampostería un sistema constructivo muy utilizado en el país, resulta necesario realizar estudios que comparen el comportamiento entre el sistema tradicional que utiliza el acero en las sisas y el que usa el acero totalmente embebido en el concreto de relleno.

Cuadro 1. Cálculo de la capacidad en cortante de muros de mampostería, según el CSCR-02 y el CSCR-10

\begin{tabular}{|c|c|c|c|c|c|}
\hline \multirow[b]{2}{*}{ Código } & \multirow{2}{*}{$\mathbf{V}_{\mathrm{s}}$} & \multicolumn{2}{|c|}{$\mathbf{V}_{\mathbf{n}}$} & \multicolumn{2}{|c|}{$\mathbf{V}_{\mathbf{m}}$} \\
\hline & & Zona de rótula plástica & Otras secciones del muro & $\begin{array}{l}\text { Acero Embebido } \\
\text { en concreto }\end{array}$ & $\begin{array}{l}\text { Acero en sisas } \\
\text { entre bloques }\end{array}$ \\
\hline 2002 & $\begin{array}{l}\mathrm{V}_{\mathrm{S}}+ \\
\mathrm{V}_{\mathrm{m}}\end{array}$ & {$\left[0.15 \sqrt{f^{\prime} m}+0.20\left(\frac{P_{u}}{A_{g}}\right)\right] d b_{w}$} & {$\left[0.50 \sqrt{f^{\prime} m}+0.30\left(\frac{P_{u}}{A_{g}}\right)\right] d b_{w}$} & \multicolumn{2}{|c|}{$\frac{A_{s h} f_{y} d}{S_{h}}$} \\
\hline 2010 & $\begin{array}{l}\mathrm{V}_{\mathrm{s}}+ \\
\mathrm{V}_{\mathrm{m}}\end{array}$ & $\left\{\left[1-0.44\left(\frac{M_{u}}{V_{u} d}\right)\right]\right.$ & $\left.+0.25\left(\frac{P_{u}}{A_{g}}\right)\right\} d b_{w}$ & $\frac{0.5 A_{s h} f_{y} d}{S_{h}}$ & $\frac{0.5 A_{s h} f_{y} d}{2 S_{h}}$ \\
\hline
\end{tabular}




\section{METODOLOGÍA}

En esta investigación primeramente se caracterizaron los materiales: morteros, concretos de relleno, unidades de mampostería y acero de refuerzo, con el fin de lograr una clase de mampostería tipo A, según la definen ambos códigos.

Se escogió un tipo de espécimen que reflejara muros de dimensiones típicas a escala natural, con la particularidad de que su patrón de falla fuera en cortante, contrario a las buenas prácticas de la ingeniería sismorresistente y a lo establecido por el CSCR-02 y el CSCR10. Es de suma importancia forzar la falla en cortante para poder establecer las verdaderas capacidades de ambos tipos de reforzamiento (acero embebido en concreto de relleno vs. acero en las sisas de los bloques).

En el Cuadro 2 se aprecian las características de los muros ensayados. NVB corresponde a los especímenes con el acero entre las sisas, y VB corresponde a los muros con el acero embebido en concreto. De cada tipo de muro considerado se ensayaron dos especímenes con las dimensiones que se indican en el Cuadro 2.

El refuerzo tanto vertical como horizontal de los muros fue el mismo para todos los especímenes. En la Figura 1 se puede apreciar esquemáticamente una elevación y un corte de los muros que se ensayaron, y la ubicación y cantidad de refuerzo que se implementó para las distintas pruebas.

Es importante destacar, que para el acero horizontal se utilizaron barras \#3 grado 40 con ganchos de $90^{\circ}$ en sus extremos, y para el vertical se utilizaron barras \#5 grado 60, con ganchos de $180^{\circ}$ en la parte superior y ganchos de $90^{\circ}$ en la parte inferior. Además, el tipo de mampostería empleado fue el tipo A.

En la fase experimental se determinaron, a partir de pruebas de materiales basadas en las normas ASTM C136, ASTM C128, ASTM C33 y ASTM 1019, los diseños de mezcla para los especímenes. Además, en esta etapa se realizó el levantamiento de los muros y se realizaron pruebas del concreto, bloques y mortero, con el material usado en la construcción de las paredes. Esto con el fin de verificar que lo planteado se apegara a lo representado en los modelos experimentales.

En la Figura 2 se muestra el montaje y sus partes, realizado con base en la norma ASTM E2126.

La carga fue aplicada mediante un gato hidráulico colocado en el muro fuerte, el cual se conectó a la viga de carga, la cual transmitió la fuerza a las paredes. El procedimiento de carga utilizado fue el dado en el método B de la norma antes citada, el cual consiste en un procedimiento de carga controlado por desplazamientos, e incluyó ciclos de desplazamientos agrupados en fases incrementando gradualmente los niveles de desplazamiento.

Cuadro 2. Características de especímenes ensayados

\begin{tabular}{ccc}
\hline & Características & \\
Muro & NVB & VB \\
Longitud & $3200 \mathrm{~mm}$. \\
Altura & $2400 \mathrm{~mm}$. \\
Tipo de bloques & $120 \times 200 \times 400 \mathrm{~mm}$. \\
Ubicación del refuerzo horizontal & En sisas & Embebido en concreto \\
\hline
\end{tabular}




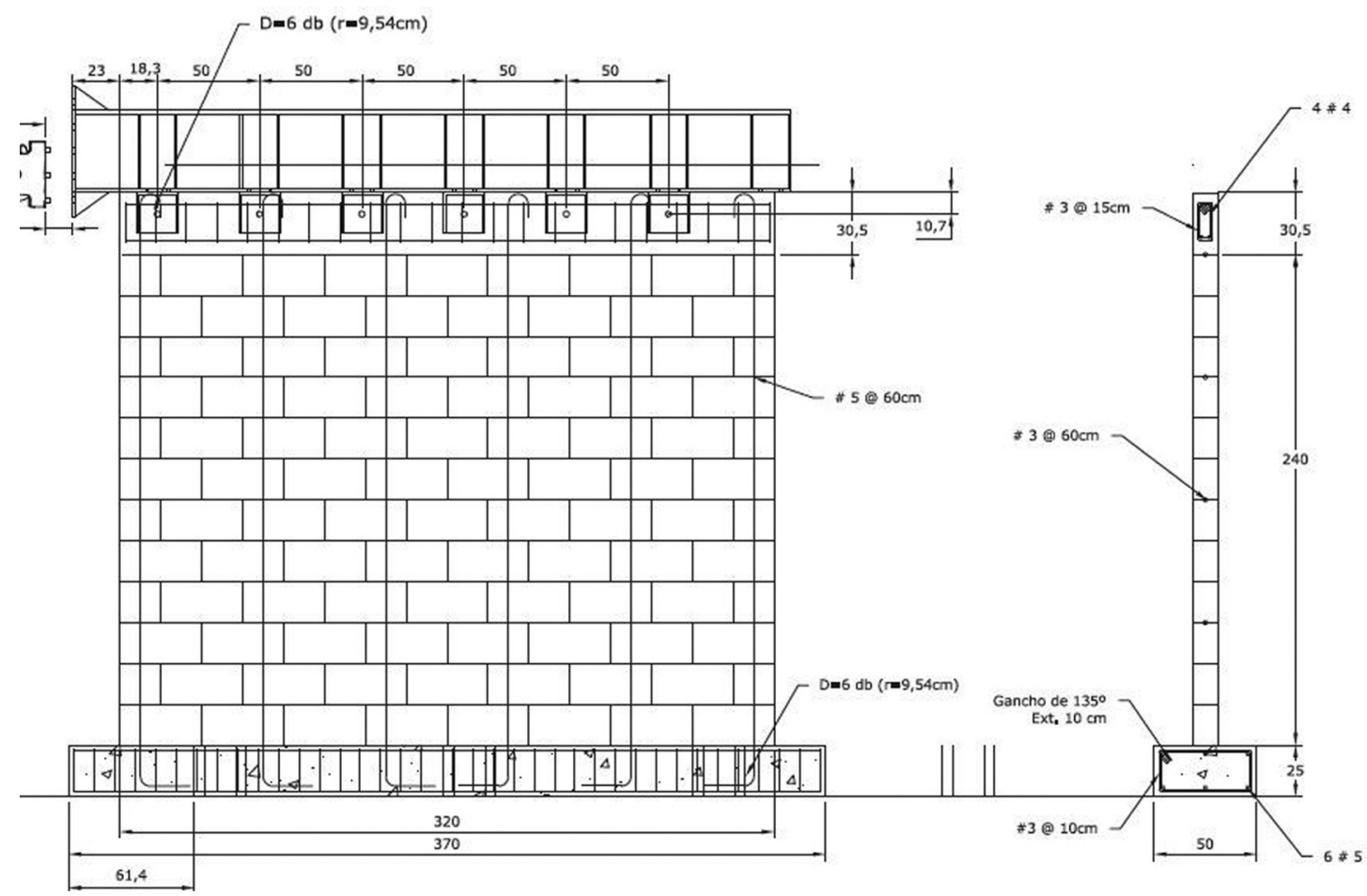

Figura 1. Detalle de paños de mampostería. Fuente: Cordero, 2012.
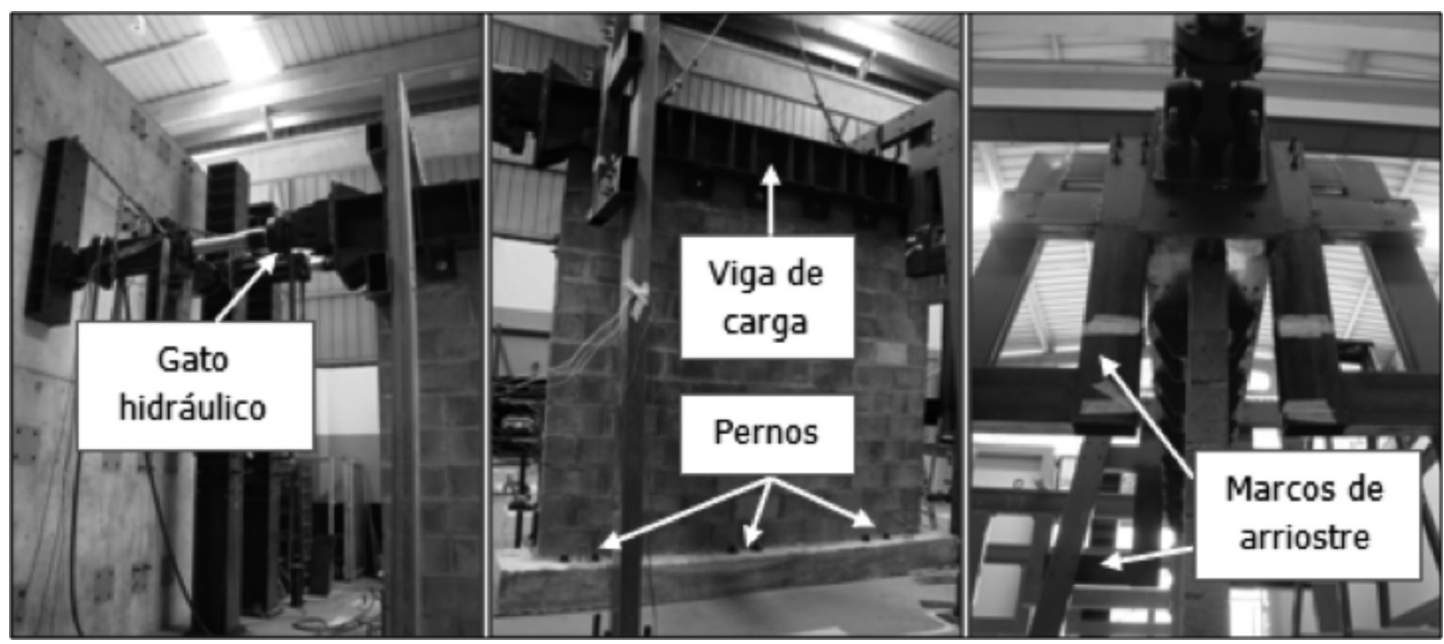

Figura 2. Montaje de pruebas experimentales.

Fuente: Cordero, 2012. 


\section{RESULTADOS}

A partir de las pruebas realizadas se demostró que para ambos tipos de muros con las características planteadas, no existe una diferencia apreciable entre el comportamiento en cortante de estos muros ante cargas paralelas al plano. De esta forma, se obtuvo que la capacidad de ambos tipos de muros es similar, y que incluso mostraron una capacidad de disipación de energía que no varió significativamente.
En el Cuadro 3 se muestran los resultados teóricos de la resistencia nominal al cortante según ambos códigos. Además, se muestran estos resultados calculados con las propiedades teóricas de los materiales, y con las propiedades reales de los mismos (Cuadro 4), que se obtuvieron a partir de pruebas realizadas en el laboratorio.

Los valores de resistencia nominal al cortante, calculados con las propiedades reales de los materiales, fueron los que se utilizaron como referencia para comparar los valores de resistencia al cortante de los muros obtenidos experimentalmente.

Cuadro 3. Comparación entre resistencias según propiedades de materiales

\begin{tabular}{cccc}
\hline $\mathbf{V}_{\mathbf{n}}(\mathbf{k N})$ & $\begin{array}{c}\text { Con propiedades } \\
\text { teóricas }\end{array}$ & $\begin{array}{c}\text { Con propiedades } \\
\text { reales }\end{array}$ & $\begin{array}{c}\text { Aumento con respecto a } \\
\text { propiedades teóricas }\end{array}$ \\
\hline CSCR-02 & 149 & 184 & $24 \%$ \\
CSCR-10, VB & 114 & 139 & $22 \%$ \\
CSCR-10, NVB & 93 & 112 & $20 \%$ \\
\hline
\end{tabular}

Fuente: Elaboración propia, 2012.

Cuadro 4. Propiedades de materiales utilizadas en cálculos

\begin{tabular}{ccc}
\hline Propiedad & \multicolumn{2}{c}{ Valor $\left(\mathbf{K g} / \mathbf{c m}^{2}\right)$} \\
Teórico & Real \\
\hline F y varilla \# 5 G60 & 100 & 140 \\
F y varilla \# 3 G40 & 4200 & 5200 \\
\hline
\end{tabular}


A continuación se muestran los resultados experimentales obtenidos para cada muro ensayado. El primer espécimen correspondió a uno de los muros con el acero entre las sisas.

En la Figura 3 se puede apreciar que el agrietamiento, a pesar de ser inclinado y en ángulos de $45^{\circ}$ aproximadamente, no fue el típico ya que las grietas no llegaron a cruzarse. Esto se debió a imperfecciones de los muros y a problemas con el montaje. Por esta razón, los resultados obtenidos a partir de este espécimen no son confiables ya que distan de los demás valores obtenidos.

A partir de la curva histerética obtenida, que se aprecia en la Figura 4, se obtuvieron los valores que se muestran en los Cuadros 5 y 6 . Es necesario mencionar que, para este ensayo, no se tomó en cuenta la parte negativa de la curva histerética por la distorsión que presentó.

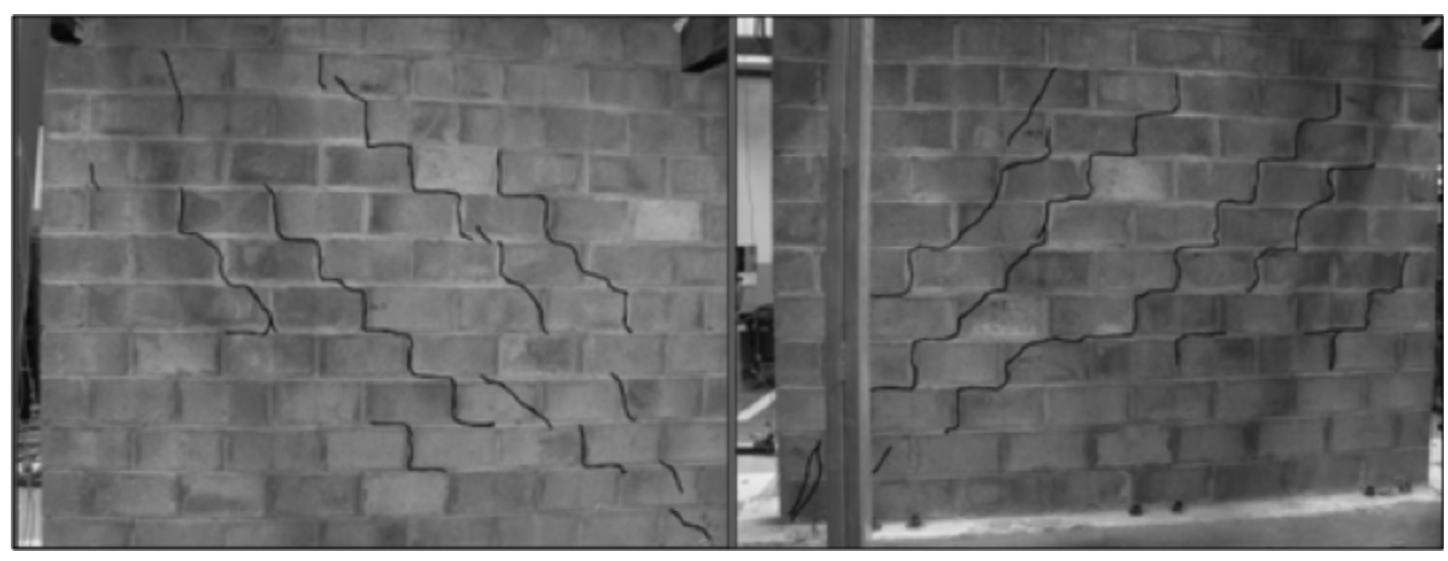

Figura 3. Patrón de grietas en NVB1.

Fuente: Cordero, 2012.

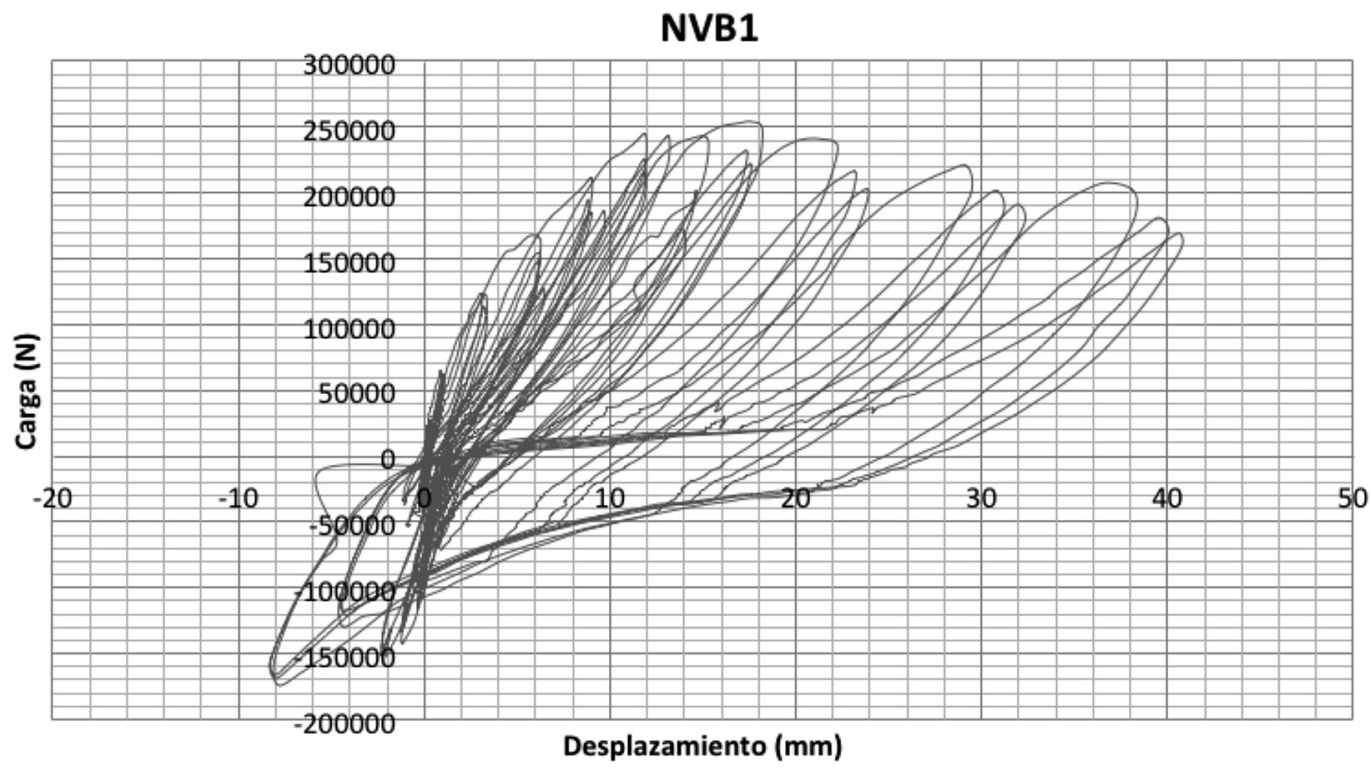

Figura 4. Curva histerética para la pared NVB1.

Fuente: Cordero, 2012. 
Cuadro 5. Resultados obtenidos a partir de curvas envolventes, pared NVB1

\begin{tabular}{ccccccc}
\hline \multirow{2}{*}{ Envolvente } & \multicolumn{9}{c}{ Datos } \\
\cline { 2 - 6 } & $\mathrm{P}_{\max }(\mathrm{N})$ & $\Delta_{\mathrm{m}}(\mathrm{mm})$ & $0.8 \mathrm{P}_{\max }(\mathrm{N})$ & $\Delta_{\mu}(\mathrm{mm})$ & $\mathrm{P}_{\text {ced }}(\mathrm{N})$ & $\Delta_{\text {ced }}(\mathrm{mm})$ \\
Positiva & 249802 & 17,5 & 199841 & 36,5 & 266086 & 4,1 \\
Negativa & N/A & N/A & N/A & N/A & N/A & N/A \\
\hline
\end{tabular}

Fuente: Cordero, 2012.

Cuadro 6. Parámetros obtenidos de la prueba para pared NVB1

\begin{tabular}{cccc}
\hline Parámetros & Envolvente Positiva & Envolvente Negativa & Promedio \\
\hline $\mathrm{V}_{\max }(\mathrm{N} / \mathrm{mm})$ & 78 & N/A & 78 \\
$\Delta$ & 9 & N/A & 9 \\
$\mathrm{k}_{\mathrm{e}}$ & 55512 & N/A & 55512 \\
\hline
\end{tabular}

Fuente: Cordero, 2012.

El segundo espécimen que se presenta en la Figura 5, corresponde al segundo muro con el acero entre las sisas. Este tuvo un agrietamiento normal, típico de la falla en cortante.

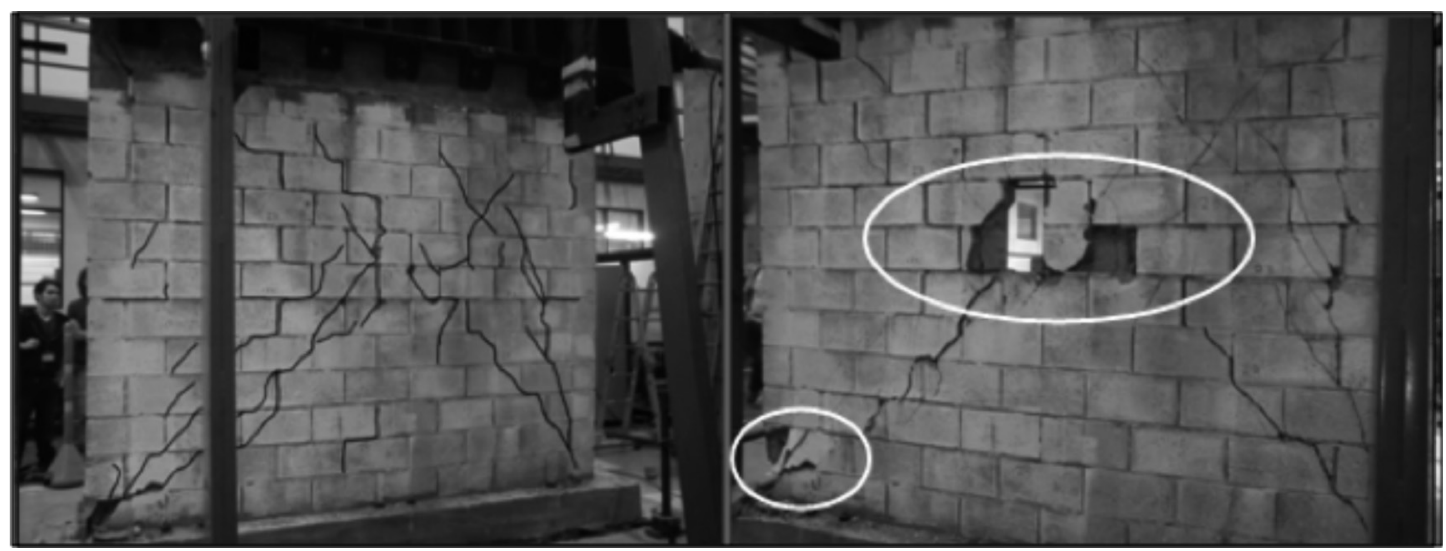

Figura 5. Patrón de grietas en NVB2.

Fuente: Cordero, 2012. 
En este ensayo, se dio una expulsión de bloques en la zona central del muro, debido a los esfuerzos generados por el agrietamiento inclinado. Además, al generarse el puntal de compresión, se afectó los bloques en uno de los extremos inferiores de la pared.

A partir de la curva histerética (Figura 6), se obtuvieron los resultados de los Cuadros 7 y 8.

Cuadro 7. Resultados obtenidos a partir de curvas envolventes, pared NVB2

\begin{tabular}{ccccccc}
\hline \multirow{2}{*}{ Envolvente } & \multicolumn{7}{c}{ Datos } \\
\cline { 2 - 7 } & $\mathrm{P}_{\max }(\mathrm{N})$ & $\Delta_{\mathrm{m}}(\mathrm{mm})$ & $0.8 \mathrm{P}_{\max }(\mathrm{N})$ & $\Delta_{\mu}(\mathrm{mm})$ & $\mathrm{P}_{\text {ced }}(\mathrm{N})$ & $\Delta_{\text {ced }}(\mathrm{mm})$ \\
Positiva & 277937 & 19,7 & 222350 & 31,0 & 235333 & 5,1 \\
Negativa & 253647 & $-17,3$ & 202918 & $-9,0$ & 153043 & 2,4 \\
\hline
\end{tabular}

Fuente: Cordero, 2012.

Cuadro 8. Parámetros obtenidos de la prueba para pared NVB2

\begin{tabular}{cccc}
\hline Parámetros & Envolvente Positiva & Envolvente Negativa & Promedio \\
\hline $\mathrm{V}_{\max }(\mathrm{N} / \mathrm{mm})$ & 87 & 79 & 83 \\
$\Delta$ & 6 & 4 & 5 \\
$\mathrm{k}_{\mathrm{e}}$ & 46323 & 63412 & 54867 \\
\hline
\end{tabular}

Fuente: Cordero, 2012.

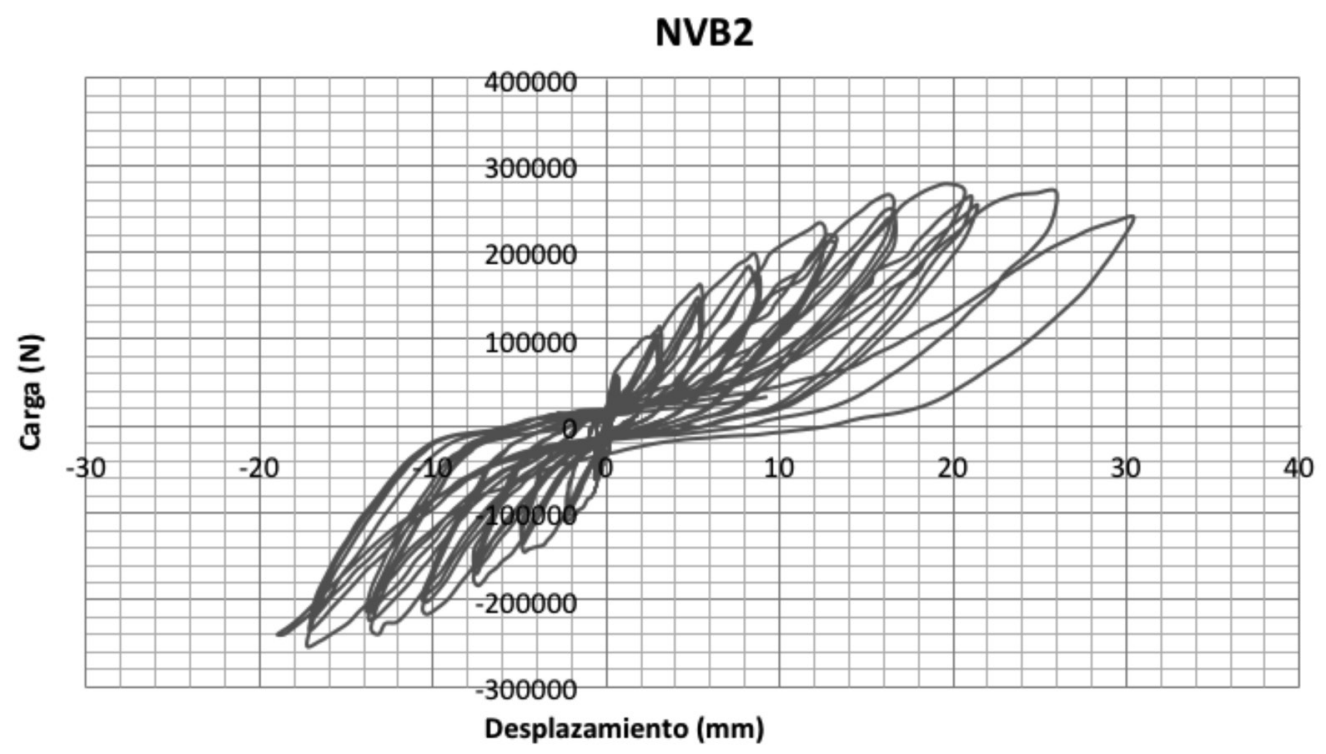

Figura 6. Curva histerética para la pared NVB2.

Fuente: Cordero, 2012. 
El tercer y cuarto especímen, Figuras 7 y 10 respectivamente, corresponden a los muros con el acero embebido en concreto. Ambos presentaron comportamientos similares en cuanto a agrietamiento, desplazamientos y cargas soportadas. Sin embargo, en estas paredes en etapas avanzadas del ensayo, se formó una grieta horizontal justo debajo de la primera hilada con el acero embebido, lo que dio origen a la expulsión de los bloques de la segunda hilada de cada espécimen (Figura 8). Los resultados para el tercer espécimen, a partir de la curva histerética mostrada en la Figura 9, se aprecian en los Cuadros 9 y 10; y los resultados del cuarto espécimen, tomados a partir de la curva histerética expuesta en la Figura 11, se muestran en los Cuadros 11 y 12.

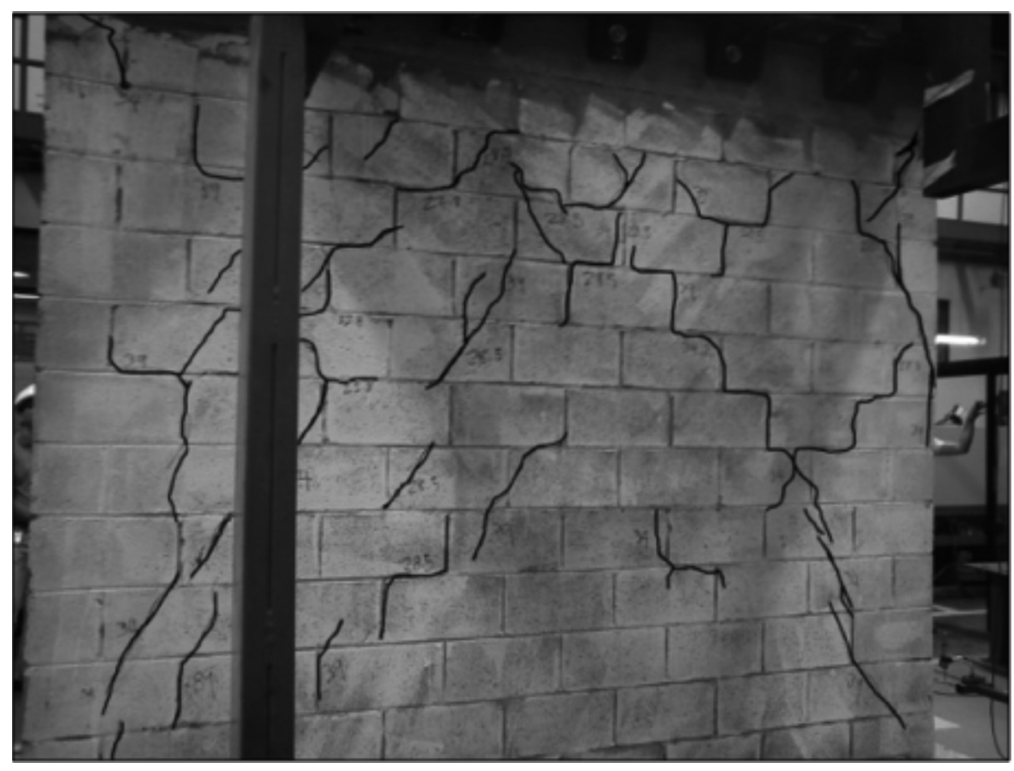

Figura 7. Patrón de grietas en VB1.

Fuente: Cordero, 2012.
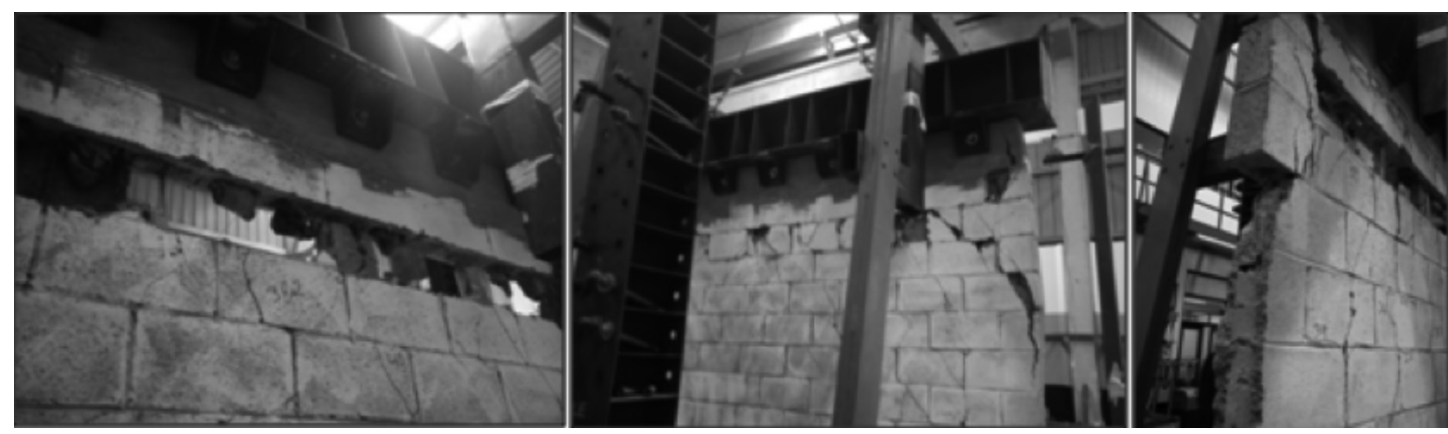

Figura 8. Expulsión de bloques bajo primera hilada en VB1.

Fuente: Cordero, 2012. 


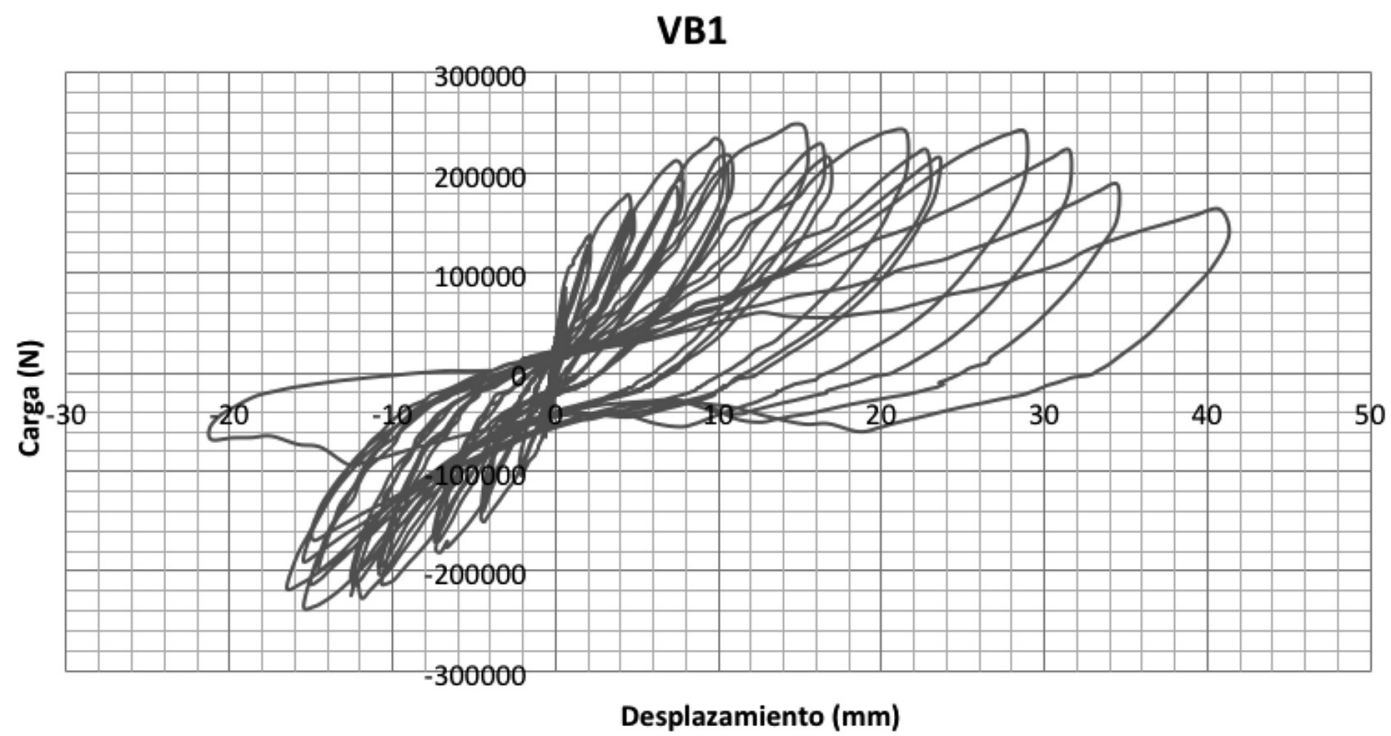

Figura 9. Curva histerética para la pared VB1.

Fuente: Cordero, 2012.

Cuadro 9. Resultados obtenidos a partir de curvas envolventes, pared VB1

\begin{tabular}{ccccccc}
\hline \multirow{2}{*}{ Envolvente } & \multicolumn{7}{c}{ Datos } \\
\cline { 2 - 7 } & $\mathrm{P}_{\max }(\mathrm{N})$ & $\Delta_{\mathrm{m}}(\mathrm{mm})$ & $0.8 \mathrm{P}_{\max }(\mathrm{N})$ & $\Delta_{\mu}(\mathrm{mm})$ & $\mathrm{P}_{\text {ced }}(\mathrm{N})$ & $\Delta_{\text {ced }}(\mathrm{mm})$ \\
Positiva & 249439 & 14,7 & 199551 & 32,0 & 243325 & 3,4 \\
Negativa & 235409 & $-15,4$ & 188327 & $-17,0$ & 175744 & $-3,2$ \\
\hline
\end{tabular}

Fuente: Cordero, 2012.

Cuadro 10. Parámetros obtenidos de la prueba para pared VB1

\begin{tabular}{cccc}
\hline Parámetros & Envolvente Positiva & Envolvente Negativa & Promedio \\
\hline $\mathrm{V}_{\max }(\mathrm{N} / \mathrm{mm})$ & 78 & 74 & 76 \\
$\Delta$ & 9 & 5 & 7 \\
$\mathrm{k}_{\mathrm{e}}$ & 71268 & 55390 & 63329 \\
\hline
\end{tabular}

Fuente: Cordero, 2012. 


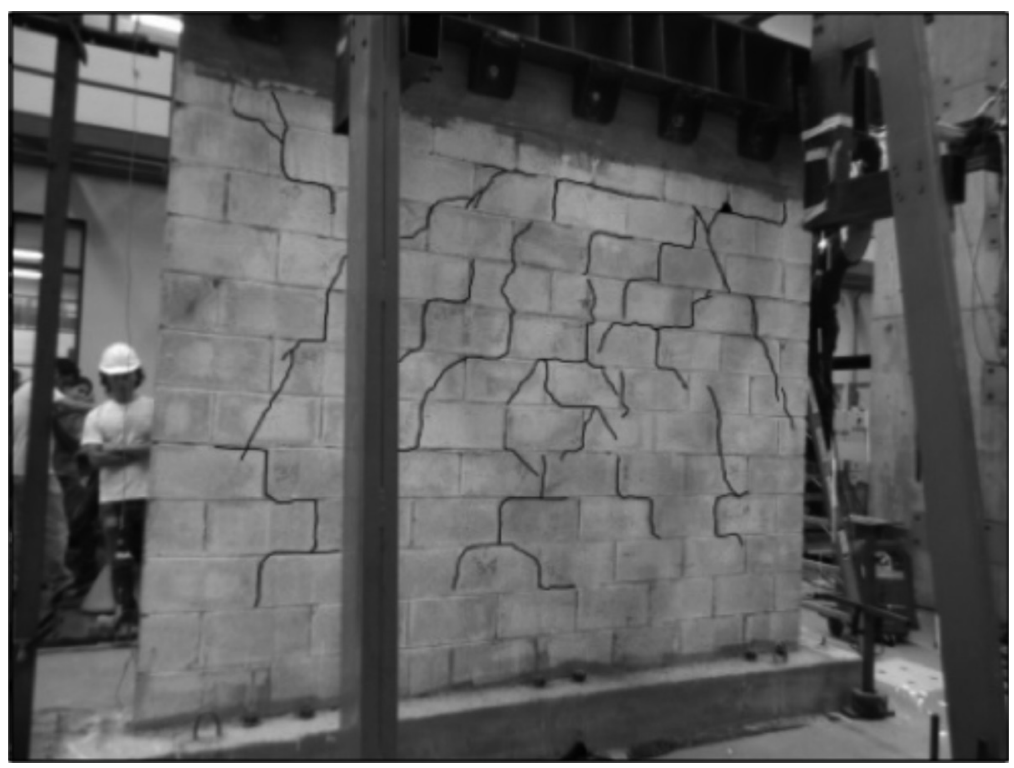

Figura 10. Patrón de grietas en pared VB2.

Fuente: Cordero, 2012.

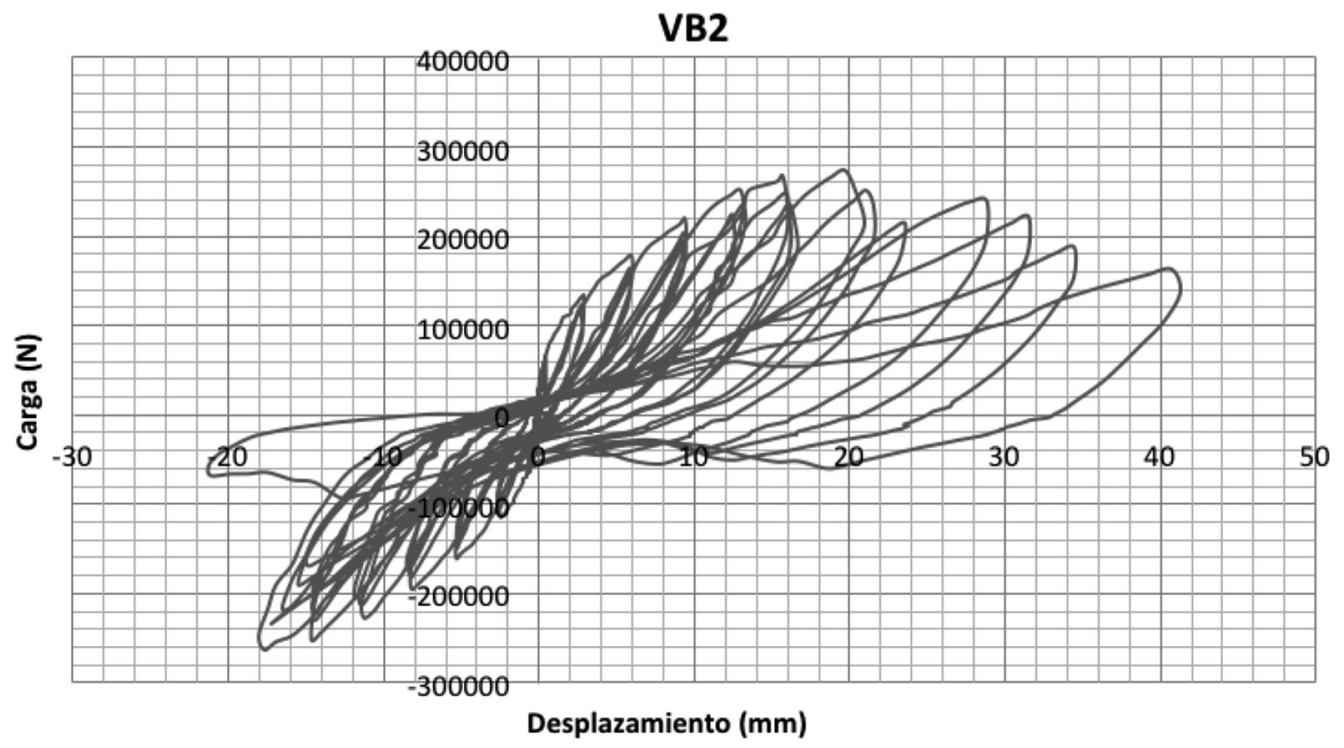

Figura 11. Curva histerética para la pared VB2.

Fuente: Cordero, 2012. 
Cuadro 11. Resultados obtenidos a partir de curvas envolventes, pared VB2

\begin{tabular}{ccccccc}
\hline \multirow{2}{*}{ Envolvente } & \multicolumn{7}{c}{ Datos } \\
\cline { 2 - 7 } & $\mathrm{P}_{\max }(\mathrm{N})$ & $\Delta_{\mathrm{m}}(\mathrm{mm})$ & $0.8 \mathrm{P}_{\max }(\mathrm{N})$ & $\Delta_{\mu}(\mathrm{mm})$ & $\mathrm{P}_{\text {ced }}(\mathrm{N})$ & $\Delta_{\text {ced }}(\mathrm{mm})$ \\
Positiva & 274533 & 19,8 & 219626 & 31,0 & 235014 & 3,2 \\
Negativa & 262722 & $-17,6$ & 210177 & $-18,5$ & 189880 & 3,4 \\
\hline
\end{tabular}

Fuente: Cordero, 2012.

Cuadro 12. Parámetros obtenidos de la prueba para pared VB2

\begin{tabular}{cccc}
\hline Parámetros & Envolvente Positiva & Envolvente Negativa & Promedio \\
\hline $\mathrm{V}_{\max }(\mathrm{N} / \mathrm{mm})$ & 86 & 82 & 84 \\
$\Delta$ & 10 & 5 & 8 \\
$\mathrm{k}_{\mathrm{e}}$ & 73209 & 55310 & 64259 \\
\hline
\end{tabular}

Fuente: Cordero, 2012.

Cuadro 13. Comparación de resultados.

\begin{tabular}{cccc}
\hline Valor & Pared NVB & Pared VB & Diferencia \\
\hline $\mathrm{P}_{\text {ced }}(\mathrm{N})$ & 210137 & 210991 & $0 \%$ \\
$\mathrm{P}_{\max }(\mathrm{N})$ & 257797 & 255525 & $1 \%$ \\
$\Delta_{\text {ced }}(\mathrm{mm})$ & 3,9 & 3,3 & $15 \%$ \\
$\Delta_{\mathrm{m}}(\mathrm{mm})$ & 18,0 & 16,9 & $6 \%$ \\
$\Delta_{\mu}(\mathrm{mm})$ & 28,3 & 24,6 & $13 \%$ \\
$\mathrm{~V}_{\max }(\mathrm{N} / \mathrm{mm})$ & 81 & 80 & $1 \%$ \\
$\Delta$ & 6,9 & 7,4 & $-7 \%$ \\
$\mathrm{k}_{\mathrm{e}}$ & 55189 & 63794 & $-16 \%$ \\
\hline
\end{tabular}

Fuente: Cordero, 2012.

A partir del promedio de los resultados obtenidos en los ensayos para ambos tipos de muro se obtuvo el Cuadro 13, en el cual se realiza una comparación de los datos.

Se compararon los resultados obtenidos experimentalmente contra los obtenidos de forma teórica con base en las ecuaciones del CSCR-02 y CSCR-10. De esta forma se obtuvo la información que se presenta en el Cuadro 14 y 15 respectivamente, y se grafica en las Figuras 12 y 13.

Por último, con la información obtenida de los ensayos se calculó la disipación de energía para cada 
Cuadro 14. Comparación entre carga soportada y resistencia teórica, CSCR-02.

\begin{tabular}{cccccc}
\hline \multicolumn{2}{c}{$\begin{array}{c}\text { Cargas máximas } \\
\text { experimentales (N) }\end{array}$} & $\begin{array}{c}\text { Resistencias teóricas } \mathbf{V}_{\mathbf{n}} \\
\text { según CSCR-02 }(\mathbf{N})\end{array}$ & & Diferencia & Diferencia \\
\cline { 1 - 2 } NVB & VB & CSCR-02 & & \\
257797 & 255525 & 183928 & $-40 \%$ & $-39 \%$ \\
\hline
\end{tabular}

Fuente: Cordero, 2012.

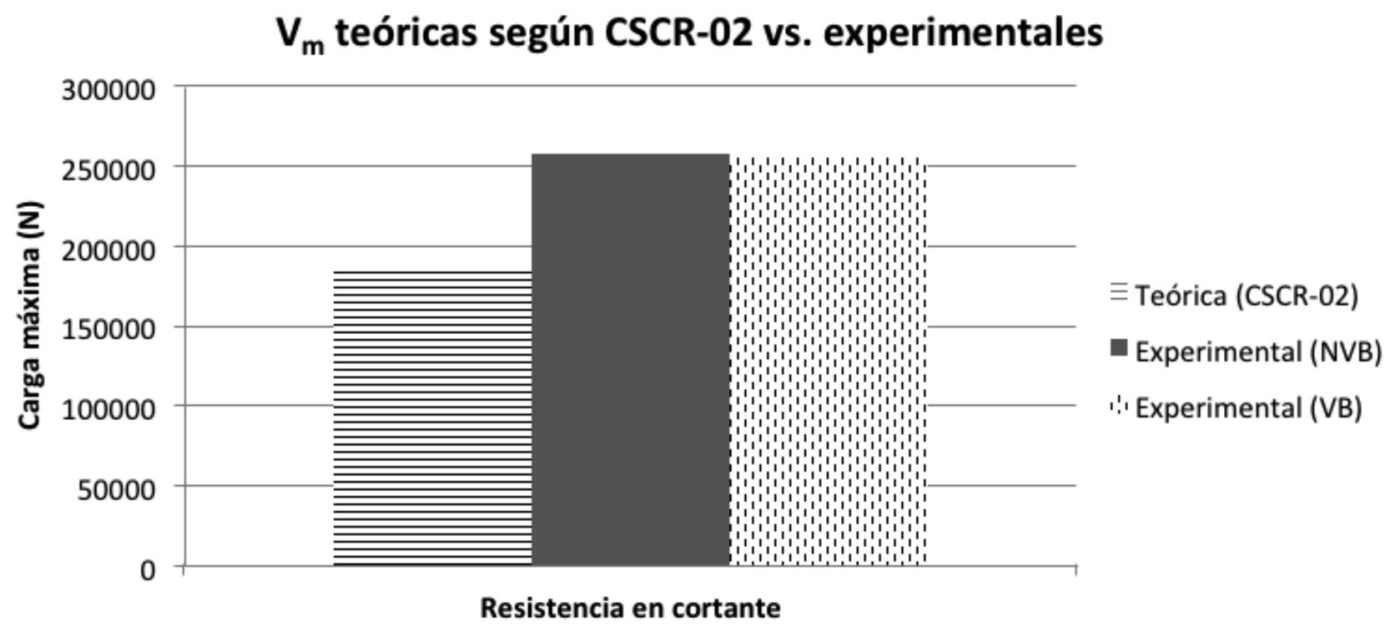

Figura 12. Comparación entre resistencias alcanzadas en ensayos y calculadas con CSCR-02.

Fuente: Cordero, 2012.

Cuadro 15. Comparación entre carga soportada y resistencia teórica, CSCR-10.

\begin{tabular}{cccccc}
\hline \multicolumn{7}{c}{ Resistencias a cortante, $\mathbf{V}_{\mathbf{n}}(\mathbf{N})$} \\
\hline \multicolumn{2}{c}{ Experimentales } & \multicolumn{4}{c}{ CSCR-10 } \\
NVB & VB & NVB & Diferencia & VB & Diferencia \\
257797 & 255525 & 112197 & $-130 \%$ & 138939 & $-84 \%$ \\
\hline
\end{tabular}




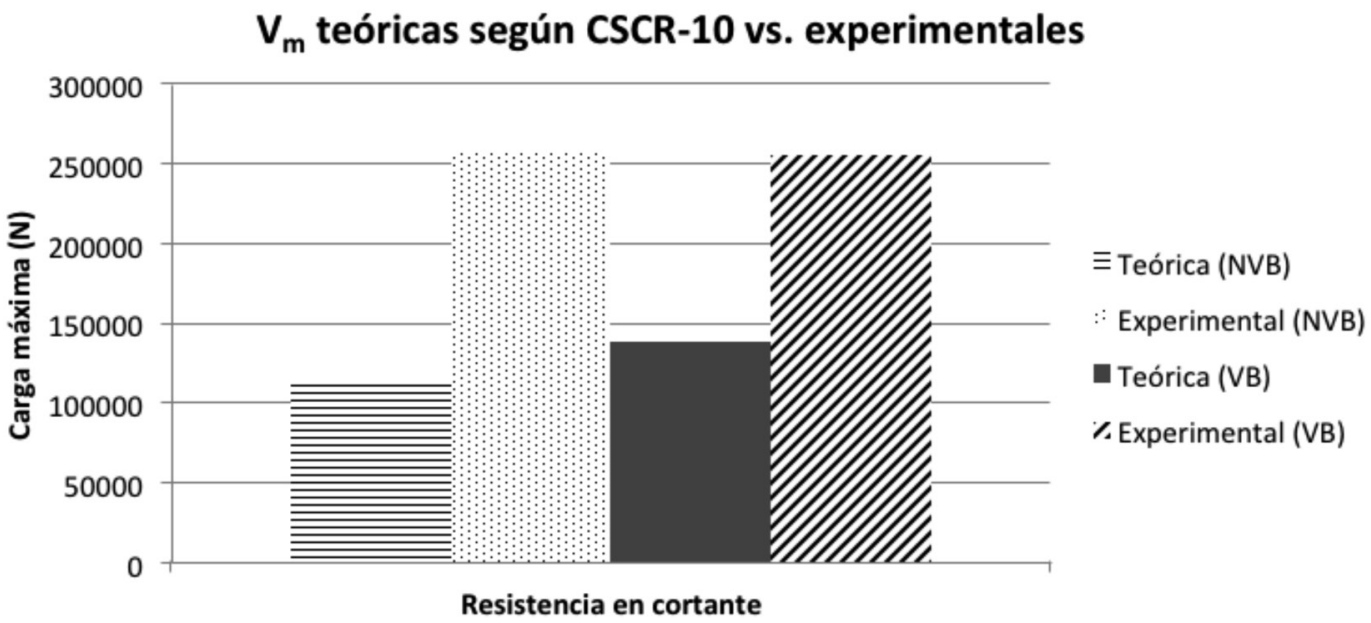

Figura 13. Comparación entre resistencias alcanzadas en ensayos y calculadas con CSCR-10.

Fuente: Cordero, 2012.

muro. Con base en las curvas histeréticas obtenidas en los ensayos se calculó el amortiguamiento para cada lazo que se muestra en la Figura 14, con el fin de obtener el amortiguamiento general para cada muro, con el cual se pudo determinar el grado de disipación de energía de cada espécimen.

Las líneas verticales que se aprecian en la Figura 14 corresponden a los desplazamientos

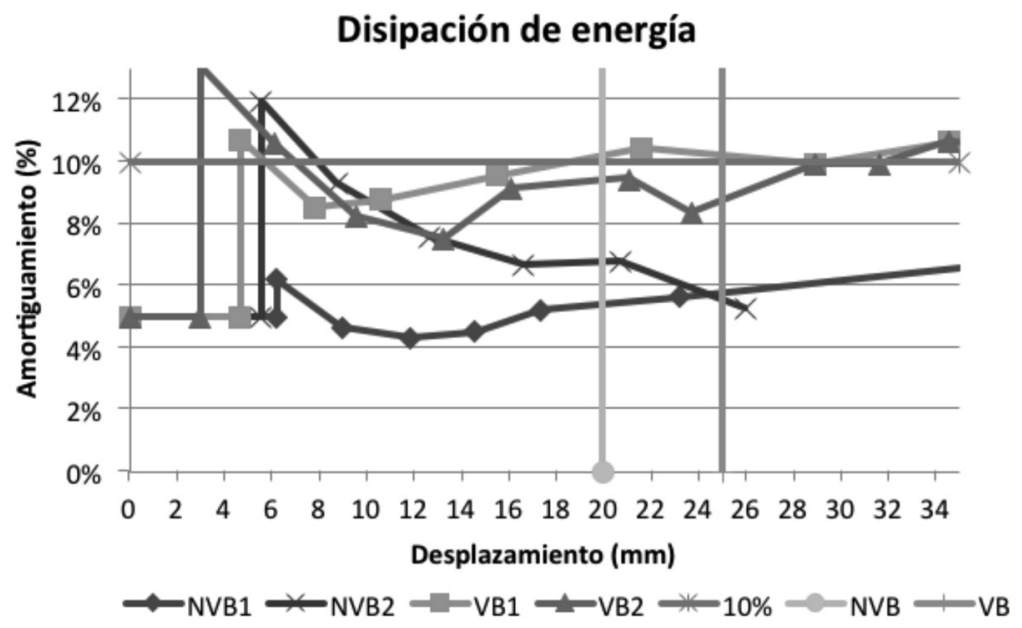

Figura 14. Disipación de energía: amortiguamiento vs. desplazamiento.

Fuente: Cordero, 2012. 
Cuadro 16. Resumen de resultados

\begin{tabular}{ccc}
\hline Especímen & Amortiguamiento & Promedio \\
\hline NVB1 & $5,3 \%$ & $6,3 \%$ \\
NVB2 & $7,2 \%$ & \\
VB1 & $8,7 \%$ & $8,9 \%$ \\
VB2 & $9,0 \%$ & \\
\hline
\end{tabular}

Fuente: Cordero, 2012.

últimos para cada tipo de espécimen. La sección horizontal al inicio de cada una de las curvas corresponde al amortiguamiento teórico de 5\% durante el rango elástico. Se graficó de esta forma, ya que durante esta fase de la prueba, los especímenes estuvieron influenciados por desplazamientos secundarios debido al acomodamiento de los mismos. Después de pasar al rango plástico, estos desplazamientos no fueron significativos y se graficó el amortiguamiento real. En el Cuadro 16 se muestra el resumen de los resultados obtenidos.

Es importante destacar, al igual que se ha hecho a lo largo del presente documento, que el valor obtenido para el espécimen NVB1 difiere mucho de su similar, por lo que se recomienda tomar en cuenta para el análisis únicamente el valor de NVB2.

\section{DISCUSIÓN}

En general, no se aprecia una diferencia significativa entre utilizar el acero embebido en concreto o colocado en las sisas de los bloques en la resistencia a cortante de los muros. Sin embargo, como únicamente tres de los cuatro especímenes pudieron ser considerados en el análisis, los resultados obtenidos no se pueden tomar como concluyentes según la estadística.

Por esta razón, se supone que los lineamientos del CSCR-10 podrían ser muy conservadores a la luz de lo obtenido, considerando muros sin empalmes y con las características ya mencionadas, por lo que se sugiere continuar con la investigación en el tema para obtener resultados más confiables.

A partir de los resultados mostrados se observa que los muros con el acero embebido en concreto de relleno presentan una rigidez elástica de cortante mayor, por lo que tienen una mayor resistencia a la deformación durante el rango elástico, lo que retardó la aparición de grietas antes de alcanzar la cedencia.

Por otro lado, se obtuvo también que la diferencia entre las cargas máximas soportadas por ambos tipos de paredes no es significativa, ya que correspondió a menos de un $1 \%$.

Los muros con el acero embebido alcanzaron un desplazamiento mayor correspondiente a un $13 \%$ de diferencia, con respecto a NVB. Este resultado es respaldado por el comportamiento observado en los muros, y por los valores de ductilidad obtenidos, los cuales son mayores para los muros VB; esto se aprecia en el Cuadro 13.

Se realizó una comparación entre los valores obtenidos con los ensayos experimentales y los calculados bajo los lineamientos del CSCR-02 y CSCR-10. En los Cuadros 14 y 15 se observa que las resistencias teóricas en cortante varían en porcentajes que van de $39 \%$ hasta $130 \%$, con respecto a las cargas reales soportadas por los muros durante las pruebas, siendo las resistencias teóricas menores a las obtenidas experimentalmente. Lo anterior se puede apreciar mejor en las Figuras 13 y 14.

Por último, con base en la Figura 14 se puede inferir para los tres últimos ensayos (NVB2, VB1, VB2), que el comportamiento en el rango plástico 
hasta alcanzar $\Delta_{\mathrm{u}}$ es muy similar, observándose sin embargo que para el muro con el acero entre las sisas, la capacidad de disipar energía disminuye más rápidamente al avanzar el desplazamiento.

\section{CONCLUSIONES}

Con base en el análisis de los resultados obtenidos y la investigación realizada, se concluye que:

- La falla de los muros fue por cortante, lo cual era lo esperado ya que se colocó suficiente acero vertical para evitar la falla por flexión. No obstante, a pesar de ser una falla en cortante, se observó un comportamiento con alguna ductilidad donde la grieta principal diagonal se propagó lentamente hasta atravesar el acero de los extremos. Este comportamiento se dio debido a la fricción generada entre grietas y la acción de dovela del refuerzo vertical.

- El mecanismo de falla bajo cargas paralelas al plano en ambos tipos de paredes fue similar, dándose en ambos casos el patrón de grietas esperado por falla en cortante, o sea, con grietas diagonales inclinadas en ángulos de $45^{\circ}$ aproximadamente.

- $\quad$ En ambos tipos de paredes, la reversibilidad de las cargas provocó la disminución de la resistencia debido a un proceso gradual de degradación de las inercias, que implicó una disminución de la rigidez de las paredes. Este efecto se ve reflejado en las curvas histeréticas de cada muro y en los cambios de pendiente de las mismas a lo largo del ensayo.

- Los muros con el acero horizontal totalmente embebidoen concreto presentaron una rigidez mayor, lo que implicó que para la misma carga presentaran menores desplazamientos, y generó que las grietas en estas paredes se dieran en ciclos más avanzados de la prueba. No obstante, después de que comenzara el agrietamiento, ambos muros alcanzaron cargas máximas similares. Esto debido a que no hubo diferencia significativa en cuanto a resistencia al variar la colocación del acero horizontal.
- Para los dos tipos de paredes estudiados se obtuvieron curvas histeréticas con comportamientos teóricos esperados; en las zonas positivas los muros alcanzaron mayores cargas con desplazamientos menores, en contraposición a la parte negativa, donde, por el efecto Baushinger, se tuvieron menores cargas, ambos después de pasar el rango elástico.

- Los muros con el acero horizontal totalmente embebido en concreto de relleno presentaron una ductilidad mayor a la obtenida en los muros con el acero en las sisas. Sin embargo, a pesar de la diferencia en las ductilidades entre ambos tipos de paredes, se obtuvo que la disipación de energía es muy similar, por lo cual no hay una ventaja significativa al utilizar un sistema o el otro en este aspecto.

- Para poder desarrollar un comportamiento de armadura en la mampostería, es necesario asegurar que el acero horizontal tenga la capacidad de soportar las cargas generadas en los muros sometidos a cortante.

- Al no existir diferencias significativas entre las cargas soportadas por ambos tipos de muros, en este caso no es posible definir si el acero horizontal resistió el cortante debido al anclaje o por la adherencia de las varillas, ya fuera en mortero o en concreto.

- Para muros de mampostería sometidos a cargas paralelas a su plano, con el anclaje de las varillas horizontales adecuado y sin empalmes, los resultados sugieren que la resistencia en cortante no presenta una variación significativa entre utilizar el acero horizontal en las sisas o totalmente embebido en su longitud dentro de concreto, y en ambos casos se obtuvo que los muros alcanzaron cargas mucho mayores a lo establecido en el CSCR-10.

- A partir de lo anterior se infiere también que los anclajes utilizados en los muros fueron efectivos, a pesar de ser una variación de lo que se especifica normalmente en muros de mampostería. 
- La normativa dada por el CSCR-10 para este tipo de muros, según lo obtenido a partir de los tres especímenes ensayados satisfactoriamente, se puede suponer conservadora, ya que se tienen diferencias entre la teoría y la práctica que superan el 100\%. Es importante destacar que esta conclusión se aplica, como ya se mencionó, para muros que no presenten empalmes en el acero horizontal y que cuenten con un anclaje que no permita deslizamientos de las varillas en la mampostería.

- Además, se aclara nuevamente que es necesario seguir con esta línea de investigación, y realizar pruebas para un número mayor de ensayos, con el fin de tener mayor evidencia para poder alcanzar afirmaciones que se puedan considerar como concluyentes respecto a si el CSCR-10 se puede considerar conservador o no.

\section{SIMBOLOGÍA}

$\mathrm{f}^{\prime}{ }_{\mathrm{m}}=$ resistencia en compresión de la mampostería a los 28 días, $\mathrm{kg} / \mathrm{cm}^{2}$.

$\mathrm{F}_{\mathrm{y}}=$ esfuerzo de cedencia del acero de refuerzo, $\mathrm{kg} / \mathrm{cm}^{2}$.

$\mathrm{k}_{\mathrm{e}}=$ rigidez de cortante elástica, $\mathrm{N} / \mathrm{mm}$.

$\mathrm{P}_{\text {ced }}=$ carga experimental de cedencia, $\mathrm{N}$.

$\mathrm{P}_{\max }=$ carga experimental máxima, $\mathrm{N}$.

$\mathrm{V}=$ fuerza en cortante, $\mathrm{N}$.

$\mathrm{V}_{\mathrm{m}}=$ resistencia nominal en cortante de la mampostería, $\mathrm{N}$.

$\mathrm{V}_{\max }=$ capacidad de cortante, $\mathrm{N} / \mathrm{mm}$.

$\mathrm{V}_{\mathrm{n}}=$ resistencia nominal en cortante, $\mathrm{N}$.

$\Delta_{\text {ced }}=$ desplazamiento en carga de cedencia, $\mathrm{mm}$.

$\Delta_{\mathrm{m}}=$ desplazamiento en carga máxima, $\mathrm{mm}$.

$\Delta_{\mathrm{u}}=$ desplazamiento en carga última, $\mathrm{mm}$.

$\varepsilon=$ amortiguamiento, $\%$.

$\mu=$ factor de ductilidad cíclico.

\section{REFERENCIAS BIBLIOGRÁFICAS}

ASTM (American Society for Testing and Materials). (2008). Standard Specification for Concrete Aggregates. ASTM C33 Philadelphia: ASTM International.

ASTM (American Society for Testing and Materials). (2011). Standard Test Methods for Cyclic (Reversed) Load Test for Shear Resistance of Vertical Elements of the Lateral Force Resisting Systems for Buildings. ASTM E2126-11 Philadelphia: ASTM International.

ASTM (American Society for Testing and Materials). (2008). Standard Test Method for Density, Relative Density (Specific Gravity), and Absorption of Fine Aggregate. ASTM C128.Philadelphia: ASTM International.

ASTM (American Society for Testing and Materials). (2008). Standard Test Method for Sampling and Testing Grout. ASTM C1019. Philadelphia: ASTM International.

ASTM (American Society for Testing and Materials). (2008). Standard Test Method for Sieve Analysis of Fine and Coarse Aggregates.ASTM C136. Philadelphia: ASTM International.

Cordero, M. (2012).Comportamiento en cortante de muros de mampostería con el refuerzo horizontal embebido en concreto o colocado en las sisas de los bloques, Proyecto de Graduación para optar por el grado de Licenciatura en Ingeniería Civil, Escuela de Ingeniería Civil, Universidad de Costa Rica, San José, Costa Rica.

Comisión Permanente de Estudio y Revisión del Código Sísmico de Costa Rica (2002). Código Sísmico de Costa Rica 2002. Cartago: Editorial Tecnológica de Costa Rica.

Comisión Permanente de Estudio y Revisión del Código Sísmico de Costa Rica (2002). Comentarios al Código Sísmico de Costa Rica 2002. Cartago: Editorial Tecnológica de Costa Rica.

Comisión Permanente de Estudio y Revisión del Código Sísmico de Costa Rica (2010). Código Sísmico de Costa Rica 2010. Cartago: Editorial Tecnológica de Costa Rica.

Gamboa, S. (1997). Determinación de la resistencia al cortante en paredes de mampostería. Proyecto de graduación para optar por el grado de Magíster Scientiae, Escuela de Ingeniería Civil, Universidad de Costa Rica, San José, Costa Rica. 
Hidalgo, J. (2005). Comportamiento estructural de paredes de mampostería integral a escala reducida fabricadas con viga bloque. Proyecto de Graduación para optar por el grado de Licenciatura en Ingeniería Civil, Escuela de Ingeniería Civil, Universidad de Costa Rica, San José, Costa Rica.

Sandí, F. (1998). Cargas cíclicas aplicadas en paredes de mampostería (sistema convencional vs sistema viga bloque). Proyecto de Graduación para optar por el grado de Licenciatura en Ingeniería Civil, Escuela de Ingeniería Civil, Universidad de Costa Rica, San José, Costa Rica.

Venegas, S. (1997). Comportamiento histerético de paredes de mampostería. Proyecto de Graduación para optar por el grado de Licenciatura en Ingeniería Civil, Escuela de
Ingeniería Civil, Universidad de Costa Rica, San José, Costa Rica.

\section{SOBRE LOS AUTORES}

\section{Alejandro Navas Carro}

M.Sc. Ingeniería Civil, Profesor de la Escuela de Ingeniería Civil de la Universidad de Costa Rica. Director del Laboratorio Nacional de Materiales y Modelos Estructuras (LANAMME). Apartado Postal 2060, UCR, San José, Costa Rica Teléfono: 2511-4186, Facsímil: 2511-4440

Correo electrónico: alejandro.navas@ucr.ac.cr

\section{Mariela Cordero Segura}

Lic. Ingeniería Civil, Ingeniera Estructural en la empresa Sismo Consultores S.A. Celular: 8836-9118 Correo electrónico: mariela_mcs@hotmail.com 